Reprod. Nutr. Dévelop., 1983, 23 (1), 81-92.

\title{
Evolution avec l'âge de la cholestérolémie et de la triglycéridémie postprandiales chez le veau préruminant ; influence de l'ingestion de sorbitol
}

\author{
D. BAUCHART
}

Avec la collaboration technique de Françoise DUBOISSET, Marinett MEYER, C. LÉOTY et R. SOUCHET

Laboratoire d'Etude du Métabolisme énergétique, I.N.R.A. Theix, 63110 Beaumont, France.

Summary. Age changes in the profiles of postprandial cholesterolemia and triglyceridemia in the preruminant calf : effect of sorbitol intake.

From birth to 10 weeks of age, 7 Friesian male calves were fed milk replacers containing 21 p. 100 tallow. The calves were divided into two groups : a control group (4 calves) and a " sorbitol " group ( 3 calves) receiving sorbitol in the milk replacer $(0.6 \mathrm{~g} / 100 \mathrm{~g} \mathrm{DM})$.

At $2,3,5,7$ and 10 weeks of age, plasma concentrations of free cholesterol, cholesterol esters and triglycerides were determined in jugular blood $2 \mathrm{~h}$ before the morning meal and $1 / 2,2,3,5$ and $7 \mathrm{~h}$ after feeding.

No significant variations were observed in the pattern of postprandial estercholesterolemia whatever the age or diet. Average estercholesterolemia was significantly higher $(P<0.01)$ at $2-3$ weeks of age than at 5,7 or 10 weeks. Sorbitol supplementation decreased estercholesterolemia significantly $(P<0.01)$ when the calves were 2 weeks old, but no effect was observed later.

Similar variations in postprandial cholesterolemia were noted for both groups at all ages. Cholesterolemia was significantly higher $(P<0.05)$ during the $-2 h,+1 / 2$ hour-period than in the 2-5- hour period and rose significantly at $7 \mathrm{~h}(\mathrm{P}<0.05)$ to the same pre-feeding level. Average cholesterolemia showed the same changes with age as estercholesterolemia in both groups.

Changes in postprandial triglyceridemia were similar to those of cholesterolemia in both groups at all the ages studied. The decrease in triglyceridemia $(P<0.01) 2 \mathrm{~h}$ after feeding was probably related to intense lipolysis, and the increase $(P<0.05) 7 \mathrm{~h}$ after feeding was due to the arrival of new feed triglycerides. Triglyceridemia was significantly $(P<0.05)$ lower when the calves were 10 weeks old than when they were younger, but no change was observed when sorbitol was added to the diet.

\section{Introduction.}

Chez l'animal domestique comme chez l'Homme, la détermination des teneurs en lipides du plasma constitue un des éléments d'appréciation de l'état nutritionnel et physiologique de l'organisme.

Faible à la naissance, la lipémie augmente fortement au cours des premiers jours de la vie chez le Veau préruminant (Shannon et Lascelles, 1966 ; Noble et al., 
1975) comme chez l'Agneau préruminant (Noble et al., 1971). Par ailleurs, chez ces mêmes animaux, la lipémie est fortement influencée par la concentration (Noble et al., 1971 ; Bazin et Brisson, 1976) ou la composition en acides gras (Stewart et al., 1978 ; Bauchart et Aurousseau, 1980 ; Richard et al., 1980) des lipides de l'aliment d'allaitement. Chez le Veau préruminant, l'étude des cinétiques postprandiales a montré que la concentration des lipides du plasma varie fortement au cours de la journée et différemment suivant les classes de lipides (Bazin et Brisson, 1976 ; Bauchart et Aurousseau, 1981). Les cinétiques peuvent être modifiées par la nature (Bauchart et Aurousseau, 1980) ou le taux de lipides ingérés (Bazin et Brisson, 1976), ainsi que par la nature des protéines ingérées (Toullec et al., 1979).

D'un point de vue nutritionnel, les lipides constituent une des principales sources d'énergie pour les animaux préruminants mais l'emploi de régimes riches en lipides chez des veaux en période de finition augmenterait les risques de troubles métaboliques. L'addition dans l'aliment d'allaitement de sorbitol, polyalcool dérivé du glucose couramment utilisé chez l'Homme pour le traitement des troubles du fonctionnement hépato-biliaire, pourrait exercer un effet régulateur dans le métabolisme des lipides chez le Veau préruminant. II augmente le volume de bile excrétée et sa concentration en minéraux (Thivend, 1982a) et améliore la croissance, I'efficacité alimentaire et l'état sanitaire des veaux recevant un régime riche en lipides (Thivend, 1982b).

Dans le but de compléter nos connaissances acquises chez le Veau préruminant âgé de 3 semaines (Bauchart et Aurousseau, $1980 ; 1981$ ), nous avons étudié l'évolution de la cholestérolémie (cholestérol libre et estérifié) et de la triglycéridémie postprandiales entre les âges de 2 et 10 semaines chez des veaux préruminants recevant un aliment d'allaitement de type conventionnel ou contenant du sorbitol.

\section{Matériel et méthodes.}

Animaux et aliments. - Sept veaux mâles de race frisonne, choisis dans deux lots de quinze animaux pour leurs caractéristiques semblables (âge, poids), ont reçu pendant une période de 10 semaines depuis l'âge de 1 jour, à raison de deux repas égaux par jour, un aliment d'allaitement comportant notamment 65 p. 100 de poudre de lait écrémé et 21 p. 100 de matières grasses sous forme de suif.

Les animaux étaient répartis en deux lots expérimentaux, quatre recevant l'aliment témoin (lot T) et trois recevant le même aliment supplémenté en sorbitol à raison de $0,6 \mathrm{p} .100$ de la matière sèche (lot So). La composition centésimale des deux aliments est donnée dans le tableau 1.

Pour l'ensemble de la période d'étude, le gain de poids vif quotidien s'élevait à $809 \pm 66 \mathrm{~g}$ et la consommation journalière de matière sèche à $1136 \pm 42 \mathrm{~g}$ pour les animaux du lot $\mathrm{T}$; pour les animaux du lot So, les valeurs correspondantes étaient de $851 \pm 84$ et $1150 \pm 44$. L'évolution des performances des animaux est indiquée dans le tableau 2.

Prélèvements de sang. - Des prélèvements de sang ont été effectués sur chacun des veaux des deux lots aux âges de 2, 3, 5, 7 et 10 semaines aux temps 
TABLEAU 1

Composition des aliments d'allaitement.

\begin{tabular}{lcc}
\hline \multicolumn{1}{c}{ Aliments } & Témoin (T) & Sorbitol (So) \\
\hline Constituants (p. 100) & & \\
Poudre de lait écrémé & 65 & 65 \\
Suif & 21 & 21 \\
Emulsifiant & 1 & 6,0 \\
Amidon & 6,2 & 4,6 \\
Poudre de lactosérum & 5,0 & 0,6 \\
Sorbitol & 0,3 & 0,3 \\
Méthionine & 1,5 & 1,5 \\
Composé minéral vitaminé & & \\
Composition chimique (p. 100 MS) & 22 & 22 \\
Matières grasses & 22 & 22 \\
Matières azotées & - & 0,6 \\
Sorbitol & & \\
\hline
\end{tabular}

TABLEAU 2

Poids vif, gain de poids vif et consommation de l'aliment des veaux aux âges correspondant aux prélèvements sanguins.

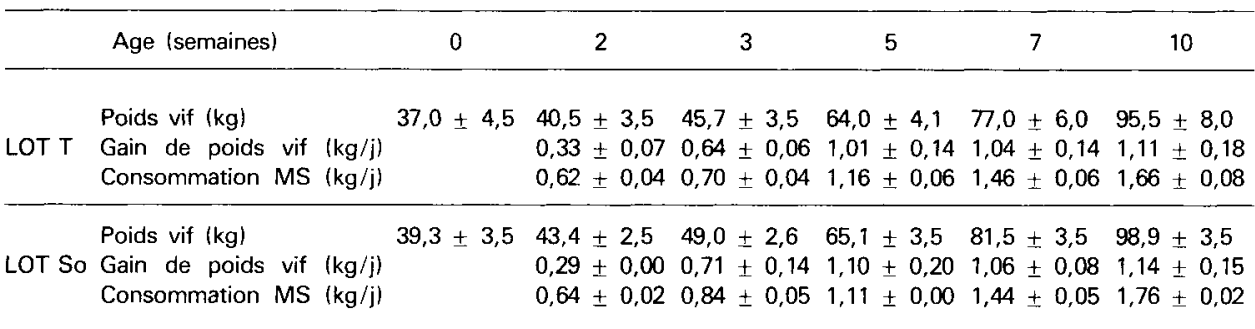

suivants : $2 \mathrm{~h}$ avant (T -2 ) puis $1 / 2$ (T1/2), 2 (T2), 3 (T3), 5 (T5) et $7 \mathrm{~h}$ (T7) après la prise du repas du matin. Les échantillons de sang $(20 \mathrm{ml})$ ont été prélevés dans la veine jugulaire, dans des tubes contenant $1 \mathrm{mg} \mathrm{d}^{\prime} E D T A / \mathrm{ml}$ de sang et immédiatement centrifugés; les plasmas récupérés ont été conservés à $-80^{\circ} \mathrm{C}$.

Analyses de sang. - Les concentrations plasmatiques en cholestérol ont été déterminées par voie enzymatique (méthode CHOD-iodure, Merckotest), respectivement avec le système cholestérol estérase-cholestérol oxydase pour le cholestérol total et le système cholestérol oxydase pour le cholestérol libre. La concentration en cholestérol estérifié a été calculée par différence entre la concentration en cholestérol total et cholestérol libre. La concentration en ester de cholestérol a été obtenue en multipliant la teneur de cholestérol estérifié par un facteur de 
1,68 ( $\left.\frac{\text { PM oléate de cholestérol }}{\text { PM cholestérol }}\right)$. La concentration plasmatique en triglycéride a été déterminée également par voie enzymatique en dosant le glycérol libéré par l'action de lipases microbiennes et transformé ultérieurement en formazan coloré (système Dow Chemical).

\section{Résultats.}

Estercholestérolémie. - Pour les deux lots expérimentaux et à tous les âges! étudiés, l'estercholestérolémie ne varie pas en moyenne significativement au cours de la période comprise entre $2 \mathrm{~h}$ avant $(T-2)$ et $7 \mathrm{~h}$ après $(T+7)$ la prise du repas (fig. 1). Pour chacun des animaux, l'estercholestérolémie postprandiale est également stable mais, entre les animaux, elle varie en moyenne de 10 à 20 p. 100.

L'estercholestérolémie moyenne, pour un âge donné, a été calculée à partir de l'ensemble des valeurs acquises au cours de la journée. Dans le lot témoin $T$, elle est élevée aux âges de 2 et 3 semaines $(109,2$ et $118,2 \mathrm{mg} / 100 \mathrm{ml}$ de plasma en moyenne) puis subit une baisse significative ( $P<0,01)$ d'environ 40 p. 100 à partir de la $5^{\mathrm{e}}$ semaine et se maintient à des valeurs non significativement différentes à 7 et 10 semaines (tabl. 3 ; fig. 1). Dans le lot supplémenté en sorbitol (lot So), l'estercholestérolémie est 2,3 fois plus faible $(P<0,01)$ que celle du lot témoin $T$ à l'âge de 2 semaines, puis dès l'âge de 3 semaines, l'évolution est la même et les concentrations ne sont plus différentes de celles du lot témoin (tabl. 3 ; fig. 1 ).

Cholestérolémie. - L'évolution postprandiale de la teneur en cholestérol libre du plasma (cholestérolémie) est significativement différente de celle de l'estercholestérolémie mais présente un profil très semblable pour les deux lots expérimentaux et à tous les âges étudiés (fig. 1). Non significativement différente $2 \mathrm{~h}$ avant et $1 / 2 \mathrm{~h}$ après le repas, la cholestérolémie baisse de 50 à $70 \mathrm{p} .1002 \mathrm{~h}$ après le repas et se maintient à une faible teneur $3 \mathrm{~h}$ et $5 \mathrm{~h}$ après le repas, puis s'élève fortement $7 \mathrm{~h}$ après le repas vers une teneur équivalente $\left(3^{\mathrm{e}}\right.$ et $5^{\mathrm{e}}$ semaines) ou supérieure $\left(2^{\mathrm{e}}, 7^{\mathrm{e}}\right.$ et $10^{\mathrm{e}}$ semaines $)$ à celle enregistrée avant le repas.

\section{TABLEAU 3}

Evolution de f'estercholestérolémie ( $\mathrm{mg} / 100 \mathrm{ml}$ de plasma) en fonction de l'âge chez le Veau préruminant (moyenne des 6 points horaires).

\begin{tabular}{lrrrrr}
\hline \multicolumn{1}{c}{ Age (semaines) } & 2 & 3 & 5 & 7 & 10 \\
\hline & $109,2 \pm 18,0^{\mathrm{a}}$ & $118,2 \pm 18,9^{\mathrm{a}}$ & $67,7 \pm 16,6^{\mathrm{b}}$ & $75,2 \pm 16,0^{\mathrm{b}}$ & $69,3 \pm 20,3^{\mathrm{b}}$ \\
Lot $\mathrm{T}$ & $47,0 \pm 21,9^{\mathrm{b}}$ & $95,6 \pm 24,1^{\mathrm{a}}$ & $80,3 \pm 20,6^{\mathrm{ab}}$ & $63,0 \pm 31,0^{\mathrm{ab}}$ & $52,3 \pm 21,0^{\mathrm{b}}$ \\
\hline
\end{tabular}

Les valeurs affectées d'une lettre différente sont significativement différentes $(P<0,01\}$. 

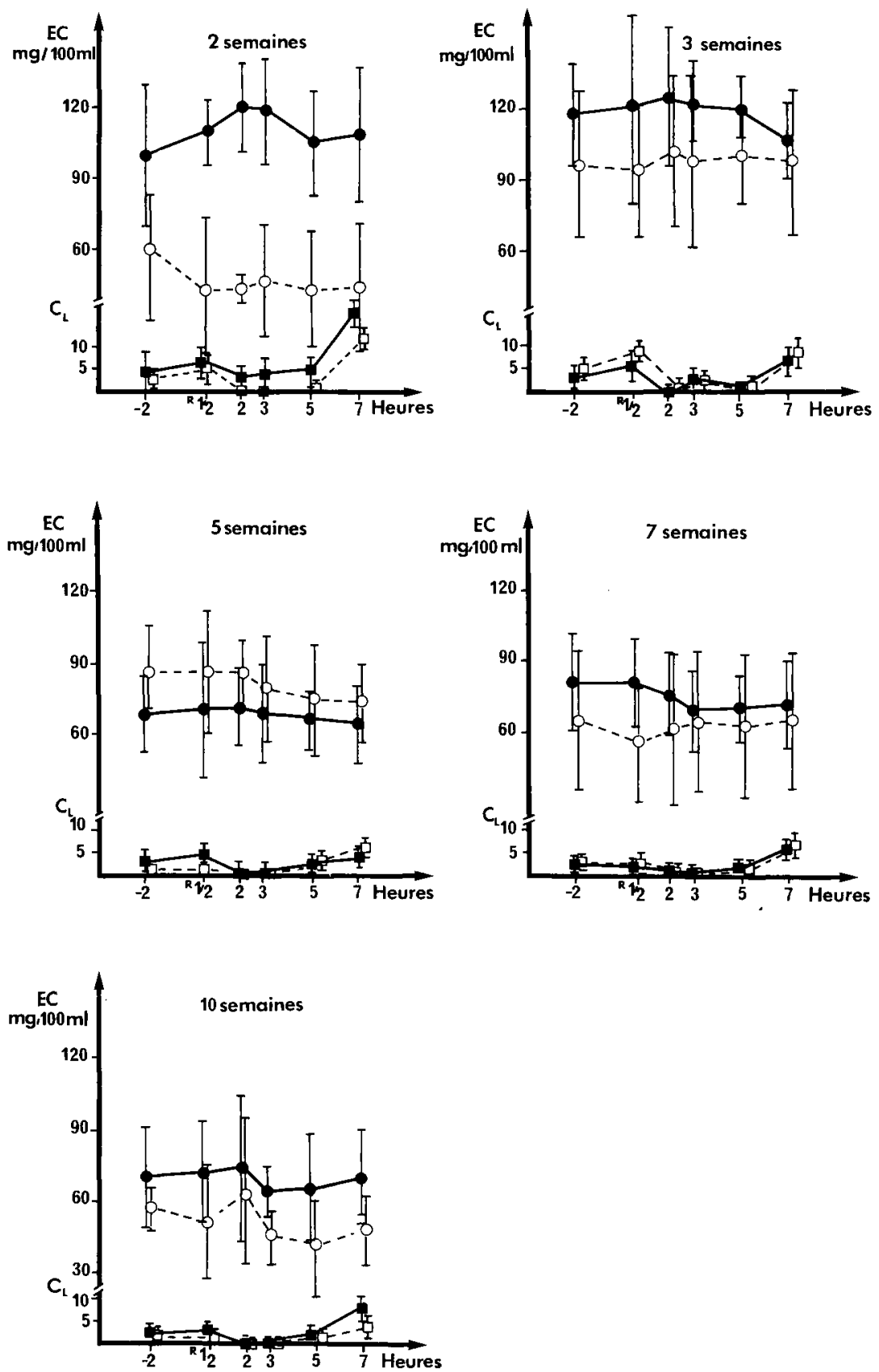

FIG. 1. - Evolution avec l'âge de l'estercholestérolémie (EC : - lot témoin; O lot "sorbitol ») et de la cholestérolémie (CL cholestérol libre : - lot témoin ; $\square$ lot " sorbitol ») postprandiales chez le Veau préruminant. 
La cholestérolémie moyenne pour un âge donné est difficile à calculer compte tenu des fortes variations des concentrations enregistrées au cours de la journée. Elle a été estimée à partir des points horaires correspondant à une période où les variations des teneurs sont les plus faibles : période a $(-2 \mathrm{~h}$; $+1 / 2 h)$ et période $b(+2 h ;+3 h ;+5 h)$. L'évolution avec l'âge de la cholestérolémie est semblable pour les 2 lots à celle de l'estercholestérolémie : les valeurs les plus élevées en période a (tabl. 4a) sont enregistrées à l'âge de 2 et 3 semaines $(3,8-7,1 \mathrm{mg} / 100 \mathrm{ml}$ plasma); ensuite, la cholestérolémie diminue significativement $(P<0,05)$ à partir de la $5^{\mathrm{e}}$ semaine (lot So : baisse de $80 \mathrm{p} .100$ ) oú de la $7^{\mathrm{e}}$ semaine (lot $\mathrm{T}$ : baisse de $58 \mathrm{p}$. 100) et se maintient à la $10^{\mathrm{e}}$ semaine. En période $\mathrm{b}$, on observe la même tendance, les teneurs ne semblent baisser qu'à partir de la $7^{\mathrm{e}}$ semaine (baisse de 76 p. 100 non significative ; tabl. 4b). Comme dans le cas de l'estercholestérolémie, le sorbitol provoque par rapport au lot témoin un abaissement significatif de la cholestérolémie à l'âge de 2 semaines (période $b, P<0,05$; tabl. $4 b$ ).

\section{TABLEAU 4}

Evolution de la cholestérolémie libre (mg/100 $\mathrm{ml}$ de plasma) en fonction de l'âge chez le Veau préruminant.

Période a : moyenne des points $-2 \mathrm{~h}$ et $+1 / 2 \mathrm{~h}$

Période $b$ : movenne des points $+2 h,+3 h,+5 h$

a)

\begin{tabular}{lccccc}
\hline \multicolumn{1}{c}{ Age (semaines) } & 2 & 3 & 5 & 7 & 10 \\
\hline & $5,6 \pm 2,8^{\mathrm{a}}$ & $4,5 \pm 3,7^{\mathrm{ab}}$ & $4,0 \pm 1,7^{\mathrm{ab}}$ & $1,7 \pm 0,4^{\mathrm{b}}$ & $2,9 \pm 2,0^{\mathrm{ab}}$ \\
Lot T & $3,8 \pm 1,2^{\mathrm{ab}}$ & $7,1 \pm 1,2^{\mathrm{a}}$ & $1,4 \pm 0,6^{\mathrm{b}}$ & $2,6 \pm 2,9^{\mathrm{ab}}$ & $0,6 \pm 0,4^{\mathrm{b}}$ \\
Lot So & & &
\end{tabular}

b)

\begin{tabular}{lccccc}
\hline Age (semaines) & 2 & 3 & 5 & 7 & 10 \\
\hline & $3,8 \pm 3,2^{\mathrm{a}}$ & $1,6 \pm 1,4^{\mathrm{b}}$ & $1,3 \pm 0,4^{\mathrm{b}}$ & $0,3 \pm 0,3^{\mathrm{b}}$ & $0,5 \pm 0,6^{\mathrm{b}}$ \\
Lot T & $0,4 \pm 0,4^{\mathrm{b}}$ & $1,6 \pm 1,5^{\mathrm{b}}$ & $1,2 \pm 1,6^{\mathrm{b}}$ & $0,3 \pm 0,3^{\mathrm{b}}$ & $0,3 \pm 0,3^{\mathrm{b}}$ \\
Lot So & & &
\end{tabular}

Les valeurs affectées d'une lettre différente sont significativement différentes $(P<0,05)$.

Triglycéridémie. - La triglycéridémie postprandiale présente une évolution semblable à celle de la cholestérolémie $(r=0,535)$. Elle est comparable pour les animaux des deux lots et aux cinq âges étudiés. La triglycéridémie est élevée mais non significativement différente $2 \mathrm{~h}$ avant et $1 / 2 \mathrm{~h}$ après la prise du repas (période a) ; ensuite, elle subit une très forte baisse (40 à 50 p. 100 en moyenne) significative $(P<0,05)$ pendant la période 2,3 et $5 \mathrm{~h}$ après le repas (période b) puis reprend une valeur égale $(3,5,7$ et 10 semaines) ou significativement $(\mathrm{P}<0,05)$ supérieure $(2$ semaines; lot $\mathrm{T}=+87 \mathrm{p} .100$; lot $\mathrm{So}=+32 \mathrm{p} .100)$ à celle de la période a correspondante, $7 \mathrm{~h}$ après le repas (fig. 2). 

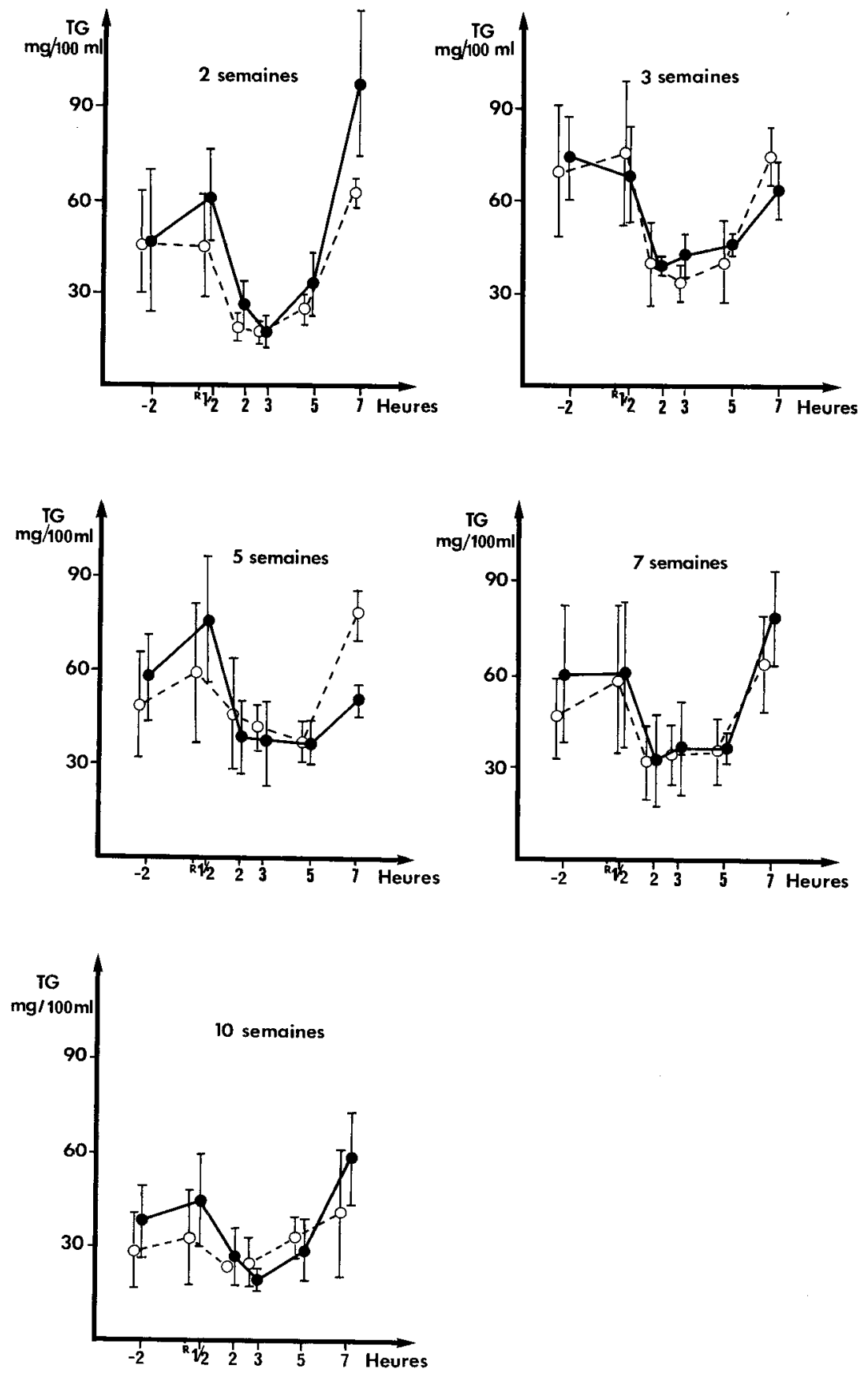

FIG. 2. - Evolution avec l'âge de la triglycéridémie ( $\bullet$ lot témoin ; O lot « sorbitol ») postprandiale chez le Veau préruminant. 
La triglycéridémie moyenne pour un âge donné est calculée, comme pour la cholestérolémie, pour les périodes a et $b$ (tabl. 5). Elle est maximale pour les deux lots à l'âge de 3 semaines (période a : $71-73 \mathrm{mg} / 100 \mathrm{ml}$ de plasma ; période b : 40-42 mg/100 ml de plasmal, comme dans le cas de l'estercholestérolémie. Elle ne varie pas ensuite significativement jusqu'à l'âge de 7 semaines puis baisse significativement pour les 2 lots $(P<0,05$ par rapport à la $3^{\text {e }}$ semaine en période $a ; P<0,05$ par rapport à la $5^{\mathrm{e}}$ semaine en période b) à l'âge de 10 semaines (tabl. 5).

TABLEAU 5

Evolution de la triglycéridémie ( $\mathrm{mg} / 100 \mathrm{ml}$ de plasma) en fonction de l'âge chez le Veau préruminant.

Période a : moyenne des points $-2 \mathrm{~h}$ et $+1 / 2 \mathrm{~h}$

Période $b$ : moyenne des points $+2 h,+3 h,+5 h$

a)

\begin{tabular}{lccccc}
\multicolumn{1}{c}{ Age (semaines) } & 2 & 3 & 5 & 7 & 10 \\
\hline Lot T & $55 \pm 17^{\mathrm{ab}}$ & $71 \pm 13^{\mathrm{b}}$ & $68 \pm 13^{\mathrm{b}}$ & $60 \pm 22^{\mathrm{ab}}$ & $42 \pm 14^{\mathrm{a}}$ \\
Lot So & $47 \pm 9^{\mathrm{ab}}$ & $73 \pm 20^{\mathrm{b}}$ & $53 \pm 27^{\mathrm{ab}}$ & $53 \pm 23^{\mathrm{ab}}$ & $30 \pm 13^{\mathrm{a}}$ \\
\hline
\end{tabular}

b)

\begin{tabular}{lccccc}
\hline \multicolumn{1}{c}{ Age (semaines) } & 2 & 3 & 5 & 7 & 10 \\
\hline & $27 \pm 6^{\mathrm{ab}}$ & $42 \pm 2^{\mathrm{c}}$ & $39 \pm 7^{\mathrm{c}}$ & $36 \pm 10^{\mathrm{ac}}$ & $25 \pm 3^{\mathrm{ab}}$ \\
Lot T & $22 \pm 4^{\mathrm{a}}$ & $40 \pm 15^{\mathrm{ac}}$ & $43 \pm 12^{\mathrm{c}}$ & $34 \pm 10^{\mathrm{ac}}$ & $27 \pm 5^{\mathrm{ab}}$ \\
\hline
\end{tabular}

Les valeurs affectées d'une lettre différente sont significativement différentes $(P<0,05)$.

\section{Discussion.}

L'addition de sorbitol $(0,6 \mathrm{~g} / 100 \mathrm{~g}$ MS) dans l'aliment d'allaitement entraîne au bout de 10 semaines et par rapport à l'aliment témoin une augmentation non significative du gain de poids vif des veaux. Cette tendance confirme les observations de Thivend (1982b) qui n'avait pas noté, dans des conditions expérimentales semblables aux nôtres, de différence significative chez des veaux jusqu'au poids de $115 \mathrm{~kg}$.

Les concentrations plasmatiques en esters de cholestérol ne présentent pas dans nos conditions expérimentales de variations significatives (malgré une légère augmentation pendant la période $2 \mathrm{~h}-5 \mathrm{~h}$ ) au cours de la journée. Cette caractéristique est remarquablement constante à tous les âges étudiés $(2,3,5,7$ et 10 semaines) et pour les deux lots expérimentaux. Les résultats sont globalement voisins de ceux obtenus chez le Veau âgé de 3 semaines par Bazin et Brisson (1976) et Bauchart et Aurousseau (1981), ces auteurs observant cependant une élévation significative mais ponctuelle $5 \mathrm{~h}$ après le repas. 
La présence de sorbitol dans I'aliment d'allaitement ne modifie pas I'allure des cinétiques postprandiales mais diminue, comme chez l'Homme recevant du sorbitol par infusion intraveineuse (Berg, Matzkies et Bergner, 1973; Berg et Matzkies, 1975), la concentration plasmatique en cholestérol libre et estérifié chez les veaux âgés de 2 semaines. Le sorbitol pourrait à cet âge soit accélérer le prélèvement du cholestérol par le foie et les tissus extrahépatiques, soit plus vraisemblablement limiter la synthèse de novo par les tissus; en effet, le sorbitol, par ses propriétés anticétogènes (Bassler et Stein, 1967), réduirait très fortement chez l'Homme le taux de $\beta$-hydroxybutyrate (Berg et Matzkies, 1975), précurseur privilégié des stérols au niveau notamment de la peau et du cerveau (Edmond, 1974).

L'évolution avec l'âge de l'estercholestérolémie moyenne marquée, chez le Veau préruminant comme chez l'Agneau préruminant (Leat, 1967; Leat et al., 1976), par un maximum à l'âge de 3 semaines et une baisse progressive pendant les 7 semaines suivantes pourrait être directement influencée par le plan d'alimentation. En effet, la forte augmentation de la vitesse de croissance à partir de la $5^{\mathrm{e}}$ semaine (gain de poids vif $>900 \mathrm{~g} / \mathrm{j}$ ) entraîne vraisemblablement une accélération du métabolisme des esters de cholestérol limitant ainsi leur concentration dans le sang alors que, chez des veaux recevant le même aliment mais en quantité limitée jusqu'à 14 semaines (vitesse de croissance inférieure à $500 \mathrm{~g} / \mathrm{j}$ ), la teneur en cholestérol total reste égal ou supérieure à la valeur observée à l'âge de 3 semaines (Barrows et al., 1980).

La teneur en cholestérol libre du plasma, faible chez le Veau préruminant ( $<10 \mathrm{mg} / 100 \mathrm{ml}$ en moyenne), évolue fortement au cours de la journée mais reste peu influencée par l'addition de sorbitol dans l'aliment d'allaitement sauf à 2 semaines où elle est significativement plus faible. Présent essentiellement dans les lipoprotéines de basse (LDL) et très basse densité (VLDL et chylomicrons) (Forte et al., 1981 ; Bauchart et Levieux, 1982), le cholestérol libre serait, quel que soit l'âge étudié, fortement estérifié au cours de la phase intense de dégradation des lipoprotéines de très basse densité (période $+1 / 2 \mathrm{~h}-+3 \mathrm{~h}$ ) (Bauchart et Levieux, 1982), ce qui expliquerait sa très faible teneur au cours de cette même période.

L'évolution postprandiale de la triglycéridémie chez le Veau préruminant (forte teneur $2 \mathrm{~h}$ avant et $1 / 2 \mathrm{~h}$ après le repas suivie d'une forte baisse pendant une période comprise entre $2 \mathrm{~h}$ et $5 \mathrm{~h}$ après le repas puis d'une forte augmentatíon à partir de $7 \mathrm{~h}$ après le repas) a déjà été décrite préalablement chez des veaux âgés de 3 semaines (Bazin et Brisson, 1976 ; Toullec et al., 1979 ; Bauchart et Aurousseau, 1981 ; Bauchart et Levieux, 1982). Cette cinétique d'évolution postprandiale reste très semblable à tous les âges étudiés et n'est pas modifiée chez des veaux recevant le lait enrichi au sorbitol. Les triglycérides, essentiellement apportés par les lipoprotéines de très basse densité (Forte et al., 1981 ; Bauchart et Levieux, 1982) et abondants $2 \mathrm{~h}$ avant et $1 / 2 \mathrm{~h}$ après le repas sont fortement dégradés par l'action de la lipoprotéine lipase au cours de la période $+1 / 2 \mathrm{~h},+3 \mathrm{~h}$, cette hydrolyse étant stimulée par la décharge d'insuline consécutive à une augmentation de la glycémie (Grizard et al., 1976). L'augmentation enregistrée sept heures après le repas pourrait s'expliquer par la baisse de l'insuli- 
némie limitant probablement la dégradation des lipoprotéines de très basse densité par de la lipoprotéine lipase. On ne peut cependant pas exclure la possibilité de l'arrivée dans le sang de TG alimentaires consécutive à l'ingestion du repas du matin qui s'ajouteraient aux TG alimentaires émis à la suite du (ou des) repas précédents. Cette dernière hypothèse expliquerait les teneurs significativement plus élevées enregistrées $7 \mathrm{~h}$ après le repas comparées à celles enregistrées $2 \mathrm{~h}$ avant et $1 / 2 \mathrm{~h}$ après le repas chez les veaux âgés de 2 semaines et serait en accord avec les données acquises sur les vitesses de transit des lipides dans la lymphe chez le Veau préruminant (Shannon et Lascelles, 1967; Toullec, 1968).

Le sorbitol n'exerce pas d'effet sur la triglycéridémie moyenne des veaux pour un âge donné. Ces observations confirment celles de Berg, Matzkies et Bergner (1973) effectuées chez l'Homme adulte normal, l'infusion intraveineuse du sorbitol n'augmentant significativement la triglycéridémie que chez des sujets hyperlipémiques, obèses ou goutteux (Berg et Matzkies, 1975).

En conclusion, cette étude montre la spécificité et la grande constance avec l'âge des courbes d'évolution postprandiales de la cholestérolémie et la triglycéridémie chez le Veau préruminant. La baisse des concentrations moyennes en cholestérol et en triglycérides avec l'âge est probablement due à une accélération des métabolismes consécutive à l'augmentation de la vitesse de croissance des animaux. L'addition de sorbitol dans l'aliment d'allaitement ne modifie pas significativement (sauf à l'âge de 2 semaines) le métabolisme des lipides plasmatiques du Veau. Ce résultat conforte l'hypothèse que chez des veaux présentant une bonne vitesse de croissance et une bonne efficacité alimentaire, le sorbitol n'exercerait pas d'effet particulier sur les fonctions de l'organisme. Par contre, avec des régimes très riches en lipides et au-delà de $100 \mathrm{~kg}$, les risques de troubles métaboliques augmentent et de précieuses informations pourraient être acquisent en contrôlant au niveau sanguin et dans ces conditions expérimentales, l'orientation du métabolisme des lipides des veaux recevant un régime enrichi ou non en sorbitol.

Recu en mai 1982.

Accepté en août 1982.

Remerciements. - L'auteur tient à remercier la Société Roquette Frères (62136 Lestrem) pour son aide financière et la fourniture en aliments d'allaitement enrichis en sorbitol.

\section{Références}

BARROWS K. K., HEEG T. R., Mc GILLIARD A. D., RICHARD M. J., JACOBSON N. L., 1980. Effect of type of dietary fat on plasma and tissue cholesterol of calves. J. Nutr., 110, 335-342.

BASSLER K. H., STEIN G., 1967. Biochemische Grundlagen für Wirkungsunterschiede zwischen Sorbit und Fructose. Hoppe-Seyler's Z. Physiol. Chem., 348, 533-539.

BAUCHART D., AUROUSSEAU B., 1980. Influence du remplacement d'une partie du suif par de I'huile de coprah dans I'aliment d'allaitement sur l'évolution postprandiale des lipides plasmatiques du jeune veau préruminant. Ann. Nutr. Alim., 34, 657-676. 
BAUCHART D., AUROUSSEAU B., 1981. Postprandial lipids in blood plasma of preruminant calves. J. Dairy Sci., 64, 2033-2042.

BAUCHART D., LEVIEUX D., 1982. Lipoprotêines plasmatiques du veau préruminant len préparation).

BAZIN R. C., BRISSON G. J., 1976. Plasma lipids, ketone bodies and glucose concentrations in calves fed high and low fat milk replacer. J. Dairy Sci, 59, 1301-1035.

BERG G., MATZKIES F., 1975 . Serumlipoproteide und Ketonkörper nach intravenöser Dauerinfusion von Sorbit. Klin. Wschr., 53, 187-188.

BERG G., MATZKIES F., BERGNER D., 1973. Verhalten der Serumlipoproteide nach Langzeitinfusion von Xylit, Fruktose und Sorbit bei gesunden Männern. Klin. Wschr., 51, 1124-1125.

EDMOND J., 1974. Ketone bodies as precursors of sterols and fatty acids in the developing rat. J. Biol. Chem., 249, 72-80.

FORTE T. D., BELL-QUINT J. J., CHENG F., 1981. Lipoproteins of fetal and newborn calves and adult steer : a study of developmental changes. Lipids, 16. 240-245.

GRIZARD J., PATUREAU-MIRAND P., PION R., 1976. Utilisation d'un régime riche en produits amylacés par le veau préruminant de poids élevé : II. Influence sur l'insulinémie postprandiale. Ann. Biol. anim. Bioch. Biophys., 16, 593-601.

LEAT W. M. F., 1967. Plasma lipids of newborn and adult ruminants and of lambs from birth to wearning. J. agric. Sci., Camb., 69, 241-246.

LEAT W. M. F., KUBASEK F. D. T., BUTTRESS N., 1976. Plasma lipoproteins of lamb and sheep. Quart. J. exp. Physiol., 61, 193-202.

NOBLE R. C., STEELE W., MOORE J. H., 1971. Diet and the fatty acids in the plasma of lambs during the first eight days after birth. Lipids, 6, 26-34.

NOBLE R. C., CROUCHMAN M. L., JENKINSON D. M., MOORE J. H., 1975. Relationship between lipids in plasma and skin secretions of neonatal calf with particular reference to linoleic acid. Lipids, 10, 128-133.

RICHARD M. J., STEWART J. W., HEEG T. R., WIGGERS K. D., JACOBSON N. L., 1980. Blood plasma lipoprotein and tissue cholesterol of calves fed soybean oil, corn oil, vegetable shortening or tallow. Atherosclerosis, 37, 513-520.

SHANNON A. D., LASCELLES A. K., 1966. Changes in the concentration of lipids and some other constituents in the blood plasma of calves from birth to 6 months of age. Aust. J. biol. Sci., 19, 831-839.

SHANNON A. D., LASCELLES A. K., 1967. A study of lipid absorption in young milk-fed calves with the use of a lymphatico-venous shunt for the collection of thoracic duct lymp. Aust. $J$. biol. Sci., 20, 669-681.

STEWART J. W., WIGGERS K. D., JACOBSON N. L., BERGER P. J., 1978. Effect of various triglycerides on blood and tissue cholesterol of calves. J. Nutr., 108, 561-566.

THIVEND P., DEBARRE M., LEFEVRE J., TOULLEC R., 1982a. Influence du sorbitol sur la sécrétion biliaire chez le Veau préruminant (résultats non publiés).

THIVEND P., 1982b. Influence du sorbitol dans l'alimentation du veau de boucherie. Bull. tech. CRZV Theix, INRA (à paraître).

TOULLEC R., 1968. Absorption des matières grasses par la voie lymphatique chez le veau préruminant. Ann. Biol. anim. Bioch. Biophys., 8, 445-447.

TOULLEC R. GUILLOTEAU P., COROLLER J. Y., 1979. Influence de la cinétique d'évacuation gastrique de l'aliment sur l'absorption chez le veau préruminant. Ann. Biol. anim. Bioch. Biophys., 19, 139-142. 